\title{
Etiquetado social: un modelo de representación de la información en la blogosfera
}

\author{
Elaine Pérez Sanchidrián \\ Ministerio de Comercio Exterior - MINCEX, Cuba \\ Raúl Campos Posada \\ Gloria Elisa Campos Posada \\ Universidad Autónoma de Coahuila - UAdeC, México
}

REVIEW

\begin{abstract}
Resumen
Objetivo: El estudio aborda los aspectos teóricos conceptuales asociados al etiquetado social. Presenta las ventajas del etiquetas en diferentes plataformas de la Web 2.0. Expone algunos de los principales sitios con sistema de etiquetado social entre los que se destacan Flickr, Delicious, Technorati, entre otros. Analiza terminológicamente el uso de las etiquetas en la blogosfera como modelo de representación de la información. Métodos: La investigación tomó como métodos el análisis de contenido cualitativo para identificar el comportamiento de la bibliografía internacional sobre esta temática y el análisis métrico con el objetivo de caracterizar el uso del etiquetado social en la blogosfera. Resultados: El estudio permitió describir cualitativamente el uso de las etiquetas en los blogs y sus particularidades terminológicas asociadas a los siguientes aspectos: la creación de las etiquetas en estos espacios esta relacionada con una serie de temáticas sociales entre las que se pueden destacar: Política, Cultura, Economía, Género, Historia, Sexualidad, Discriminación, Salud, Medio Ambiente, Tecnología. Las etiquetas en estas plataformas se definen de lo general a lo particular y no existe un límite de concurrencias para su creación, generalmente los autores citan para cada post entre 4-7 etiquetas con el objetivo de difundir lo más posible sus contenidos en la comunidad. Conclusión: El presente estudio posibilita reflejar el impacto social del uso de las etiquetas en plataformas como los blogs.
\end{abstract}

Palabras-Clave

Blogs ; Blogosfera ; Web 2.0 ; Redes sociales ; Etiquetado social

\section{Social tagging: a model for representing information in the blogosphere}

\section{Abstract}

Objective: The study addresses the theoretical and conceptual aspects related to social labeling. Has the advantages of labels on different platforms of Web 2.0. Exposes some of the major sites of social labeling system including Flickr, Delicious, Technorati is destcan, among others. Terminologically analyzed using the tags in the blogosphere as a model for representing information. Methods: The research took as methods of qualitative content analysis to identify the behavior of the international literature on this subject and the metric analysis to characterize the use of social labeling in the blogosphere. Results: The study led to qualitatively describe the use of labels on blogs and their terminological particularities associated with the following aspects: creating labels in these spaces is related to a number of social issues among which we can highlight: Politics, Culture, economy, Gender, History, Sexuality, Discrimination, Health, Environment, Technology. The labels on these platforms are defined from the general to the particular and there is no limit concurrency for its creation, usually the authors cite for each post 47 labels in order to spread their content as possible in the community. Conclusions: This study enables reflect the social impact of using labels on platforms like blogs.

Keywords

Blogs ; Blogosphere ; Web 2.0; Social network ; Social labeling 


\section{Introdución}

La web 2.0 con el objetivo de contribuir a la colaboración y el intercambio de información entre los usuarios se ha convertido mediante la virtualidad en un medio que ha hecho posible la socialización del conocimiento y la existencia de diferentes soportes de información para presentar y almacenar los contenidos. Este espacio digital provee la posibilidad mediante sitios como blogs, redes sociales, wikis y herramientas como el etiquetado social y la sindicación de contenidos, la capacidad de colocar información alrededor de un tema, para fortalecer el trabajo colaborativo entre los usuarios, en aras de fomentar la participación e interacción entre los mismos.

Estos contextos permiten la integración de varios modos de comunicación en una red interactiva, como la formación de un supertexto y un metalenguaje que, integra en el mismo sistema las modalidades escrita, oral y audiovisual de la comunicación, cuyo contenido presenta grandes facilidades de organización, representación, recuperación a través de herramientas como el etiquetado social.

La asignación de etiquetas en estos contextos permiten clasificar los contenidos ya sean las entradas realizadas en los blogs, como los enlaces que estos brindan hacia otros sitios de la red de forma libre, virtualmente sin jerarquías, de una forma más fácil, rápida, comprensible y sin límites de definición.

Estos sistemas suelen llevarse a cabo en entornos compartidos que presentan una dimensión social o colectiva: los usuarios comparten sus etiquetas y recursos, generando mediante colaboración implícita un índice global de etiquetas (folksonomía), a partir de las cuales, los usuarios pueden recuperar cualquier recurso descrito por otros.

En la actualidad el crecimiento de la blogosfera y las múltiples expresiones de contenido que en ella se publican, ha resultado especialmente interesante para la creación de una representación de la realidad que se articula espontáneamente a través de las categorías y etiquetas construidas por los blogueros en función de sus gustos, preferencias, temas, tendencias y grupos. Todo ello permite la identificación de las características de los diferentes dominios de usuarios que interactúan en estas plataformas.

Atendiendo a la importancia que presenta el etiquetado social para la Ciencias de la Información como modelo de representación de la información, este ha sido estudiado desde disímiles aristas, con el propósito de lograr una comprensión cabal de estos nuevos escenarios que emergen en la web 2.0. Debido a la importancia de este tema, el impacto de su uso en plataformas como los blogs, esta investigación pretende sentar las bases teóricas de un estudio enfocado hacia el análisis de la terminología, comportamiento y utilidad de estas herramientas en este contexto con un enfoque social.

\section{Etiquetado social}

Con el crecimiento de la información en la Web a partir de la interacción constante de los usuarios sobre plataformas como los blogs, se han generado varios problemas en cuanto a la organización y representación de la información. Los esquemas clasificatorios tradicionales resultan demasiados costosos y no son capaces de adaptarse a las formas de pensar y representar la realidad de los usuarios en la web social, es por ello que se hace necesario crear nuevas herramientas de representación de la información que provean la estabilidad, flexibilidad, simplicidad y fluidez en estos medios.

Los sistemas de etiquetado social son una de las estructuras utilizadas para la organización, representación y recuperación de la información en este contexto digital y pueden ser definidos como "un nuevo modelo para la descripción mediante palabras claves libres, donde el propio usuario tiene la posibilidad de asignar a sus recursos aquellas etiquetas (metadatos) en virtud de sus intereses que le sean útiles para la recuperación" (Maldonado, 2009).

El etiquetado social mejora la gestión de la información a través de los términos que se utilizan para categorizar páginas web, fotografías, videos, textos, etc., los cuales permiten que los usuarios logren acotar su búsqueda en función de sus intereses informativos, sin necesidad de realizar grandes esfuerzos cognitivos.

Estos sistemas suelen llevarse a cabo en entornos compartidos que presentan una dimensión social o colectiva: los usuarios comparten sus etiquetas y recursos, generando mediante colaboración implícita un índice global de etiquetas (folksonomía), a partir de las cuales, los usuarios pueden recuperar cualquier recurso descrito por otros. 
Según Cañada (2006), el etiquetado social tiene dos dimensiones interrelacionadas: la personal y la colectiva. La personal es un proceso de indización o categorización de recursos cuya principal motivación es de carácter egoísta o individual: el usuario etiqueta los recursos para poder recuperarlo posteriormente. Es decir, cada usuario confecciona su propio índice personal de etiquetas (personomía) para su colección de recursos. La dimensión colectiva ocurre cuando varios usuarios comparten sus etiquetas y recursos generando un índice global de etiquetas. El conjunto (set) de etiquetas asignadas por una comunidad es lo que se conoce genéricamente como folksonomía.

Un sistema de etiquetado social permite dividir un dominio de la realidad en una serie ordenada de clases y subclases o de categorías y subcategorías. Es un sistema de catalogación por materias preecordinadas con una estructura jerárquica. Para que estos sean eficaces sus contenidos deben abarcar una información escalonada, es decir que vaya de lo general a lo particular y alcanzar todo el campo de cada materia; se requiere que las ideas sean expresadas en todos sus grados y que a su vez se permita la combinación de estas. Deben también responder a la mecánica del pensamiento formal, ser explícitos, concisos, sencillos y su notación fácil de escribir y de recordar.

Existen diferentes motivaciones para que los usuarios atribuyan etiquetas a los objetos. Estos pueden etiquetar objetos que apunten "hacia una recuperación futura donde se incita a una actividad o a simples recordatorios tanto así mismos u otros" (Gupta, 2011). Este tipo de etiquetas descriptivas son útiles proporcionando los metadatos sobre aquellos objetos que no presentan términos asociados. Estas pueden utilizarse como contribución para describir recursos o agregar el recurso a racimos conceptuales que posibiliten un valor para el público conocido y desconocido.

Estas pueden son explotadas por los internautas para "llamar la atención dentro de la comunidad y conseguir que las personas aparezcan en los propios recursos de uno" (Gupta, 2011). La necesidad de compartir con otros la expresión de una opinión y una serie de juicios es otra de las motivaciones que propician la creación de estos términos.

Estas pueden generarse también con el objetivo de organizar los contenidos o comunicar información contextual sobre el objeto a otros. En función de las motivaciones del usuario para la creación de estos términos se pueden determinar la tipología de estas.

La asignación de etiquetas como proceso intelectual, posibilita describir el contenido lo más exactamente posible a una temática, de manera que quede ser agrupado posteriormente con recursos semejantes. Su forma de representación es sintética y se relaciona con los términos del lenguaje natural.

Según Cañada (2006) existen cuatro estilos muy diferenciados para clasificar estos términos: etiquetado egoísta, amiguista, altruista y populista.

El etiquetado egoísta se determina en el uso de esas etiquetas que no presentan significado alguno para otros usuarios de la comunidad. Estas son utilizadas para consumo personal y la búsqueda de cualquier contenido que se necesite posteriormente. La construcción de estas depende de la madurez del blogger. Por su parte la construcción de aquellos términos que sirven para compartir con personas pertenecientes a un círculo de amistades más cerrado, son las que se definen como etiquetas amiguistas. Este tipo de etiquetado aporta mucho beneficio social para grupos pequeños ya que permite compartir y reforzar la pertenencia entre el grupo.

Las etiquetas cuyo propósito de creación es compartir con el resto de la comunidad bloquera, utilizando de esta manera las etiquetas más generales utilizadas por otros usuarios para describir el contenido son consideradas altruistas. Su beneficio social para la comunidad radica en facilitar la recuperación de información para otros usuarios. Por otro lado, los blogger que son capaces de generar términos con el objetivo de obtener un beneficio directo de popularidad dentro de la comunidad, utilizando términos sin significado semántico para los usuarios, se denominan populistas. Este tipo de etiquetado es completamente irrelevante durante el proceso de recuperación de la información y navegación dentro del sitio.

Estas etiquetas "no siguen ninguna pauta formal ubicua en la actualidad, lo cual significa que pueden categorizarse los artículos con cualquier palabra que defina una relación entre el recurso en línea y un concepto en la mente del usuario. Podría escogerse cualquier número de palabras, algunas de las cuales podrían tener poco sentido fuera del contexto del autor de la etiqueta, razón 
por la que el conocimiento cabal de esos usuarios, sus motivaciones, sus decisiones y sus sistemas de cooperación son imprescindibles" (Hernández, 2008).

La clasificación de la información amparada en estos sistemas de etiquetado social reporta para los usuarios las posibilidades de anotar sus enlaces junto a términos que pueden parecerle relevantes para su descripción sin que medie un lenguaje controlado, debido a que este proceso es capaz de modificar el conocimiento existente, a través de la propia práctica mediante el intercambio de información.

El etiquetado social atendiendo a sus escenarios colaborativos pueden ser capaces de identificar "acerca de qué (o quién) trata el recurso, las etiquetas identifican los asuntos de los recursos marcados. Estos recursos, son descritos mediante sustantivos de uso corriente en muchos niveles de especificidad, pero también usando nombres propios en el caso de contenidos referidos a personas o instituciones" (Golder y Huberman, 2006).

Las etiquetas pueden referir que tipo de cosa es el recurso etiquetado, independientemente de cuáles sean las materias fundamentales, identificando de esta manera que es el recurso. Algunas de estas son asignadas para identificar el sujeto creador del recurso. Por otra parte la etiqueta que no es capaz de expresar por sí sola toda la carga de significado que tiene el tema a representar por lo que se construye, solo permiten identificar los contenidos asociados a su relación con el usuario, las cuales pueden estar encaminadas hacia funciones organizativas relacionada con la realización de alguna actividad específica del creador.

El etiquetado social no se puede definir como innovador, sin embargo desde el punto de vista de la recuperación de la información, "las etiquetas brindan una dimensión social que ofrece mayores expectativas: descripción intersubjetiva, mayor exhaustividad y consistencia en la indización, y posibilidad de extraer relaciones de semántica subyacente. El proceso mental de clasificación mediante etiquetas exige menos del usuario, ya que no resulta preciso escoger opciones relevantes dentro de una lista predefinida" (Sinha, 2005).

Estas herramientas posibilitan clasificar los enlaces mediante una o varias etiquetas, crear redes o grupos de usuarios que comparten sus enlaces mediante nubes de etiquetas, lista de enlaces en sus blogs, permitiéndoles subscribirse mediante RSS a la cuenta de un usuario determinado o a etiquetas de contenidos que te interesen. La organización de grandes cúmulos de información es otra de las ventajas que brindan estos de modelos, así como el almacenamiento de los enlaces creados por otros usuarios y la edición de los mismos en línea.

La popularidad de estos medios se efectuó a través del lanzamiento de algunos sitios gratuitos de gestión de la información como: Delicious, Flirck, Technorati, etc., a través de los cuales, se fomentaron las relaciones de intercambio y colaboración en la red

Delicious, es uno de los servicios más populares, una vez que te has registrado y creado tu cuenta ya puedes empezar a crear tu lista de favoritos en línea. Este es un marcador social de sitios web y una herramienta bookmarking que permite salvar en su plataforma los enlaces a páginas web favoritas para su futura recuperación. Este sistema permite añadir etiquetas para describir el contenido de los sitios web escogidos, facilitando de esta manera su organización y recuperación en un futuro cercano de la información, así como, la utilización de estos enlaces por otros usuarios de la comunidad. Según Quintarrelli (2005) este sistema colaborativo brinda muchas funcionalidades:

1. Una base de datos compartida de recursos.

2. Un vocabulario compartido cuyo uso constituye una innovadora posibilidad para la navegación y búsqueda.

3. La posibilidad de monitorear cambios temáticos en áreas de interés individual y social.

4. Contar con novedosos mecanismos de representación y visualización de etiquetas.

Por su parte Flirck es "un gestor de imágenes digitales que permite organizar colecciones de fotos en álbumes y asignarle etiquetas descriptivas a las mismas" (Kroski, 2005).

Este sitio de red social fue lanzado en febrero del 2004 por Ludicorp, una compañía de Vancouver. Flirck es un repositorio fotográfico que permite subir fotos o imágenes digitales a través de una plataforma simple y sencilla. 
Alguna de sus características técnicas es el uso de etiquetas (tags) por los usuarios para describir las fotos publicadas, planteándolo como un sistema de indexación social, en la que los propios usuarios emplean "tags" o etiquetas de modo descentralizado sobre objetos diversos.

En Flirck las etiquetas posibilitan organizar y representar las imágenes en múltiples grupos relacionados para su recuperación, brindando así, varias funcionalidades en lo que respecta a su navegación. Este sitio permite "la conformación de grupos de usuarios. La creación de redes de amigos entre los miembros del sitio, permite a los usuarios formar grupos que comparten intereses al subir imágenes. Cuenta con novedoso mecanismos de comunicación y formas de organización social entre las que se destacan: el envío de mensajes a usuarios, comentario de fotos de interés, asignar a las imágenes niveles de preferencia o favoritismo, entre otras" (Mallow, 2006 y Kroski, 2005).

\section{Por otro lado Technorati es un sistema Web que posibilita a los usuarios" agregar blogs de su interés, realizar búsquedas en los contenidos de estos y etiquetar sus propios posts y compartirlos con la comunidad bloggers. Es considerado el mayor buscador de blogs ya que supervisa la actualización de más de 70 millones de blogs, así como de 11 millones de etiquetas entre las que se incluyen las generadas en la dinámica taggeadora de Flirck y Delicious" (Naya, 2009).}

Existen otros sitios que permiten la definición de etiquetas tal es el caso de los sitios del blogging como: WordPress, Blogger, donde los autores pueden agregar las etiquetas a sus post para posibilitar una mejor recuperación de la información. En estas plataformas los usuarios anotan a menudo partes de las fotografías y pueden proporcionar también etiquetas a la información en otros formularios como que les gustan las señales o los enlaces publicados; en los sitios de catalogación como LibraryThing y Shelfari permiten a los usuarios etiquetar los libros, en sitios de noticias sociales como Digg, SlashDot, los usuarios pueden atar las etiquetas a las historias de las noticias y los objetos multimedios como el podcasts, los lanzamientos vivos, videos y música se etiquetan en los sitios como Youtube, imeem, Metacafe, etc.

Otra aplicación de etiquetado social es Mister Wong, el portal de marcadores sociales más importante de Europa, que está disponible en varios idiomas, entre ellos el español.

Mister Wong es uno de los mayores marcadores sociales gratuitos de Europa y uno de los más usados en el ámbito educativo por su sencillez y prestaciones. La versión original de Mister Wong, en alemán, comenzó en marzo de 2006 y cuenta con más de 2.55 millones de visitas al mes. Actualmente, el servicio está disponible en alemán, inglés, ruso, chino, español y francés.

Este sistema posibilita a los usuarios guardar de forma pública o privada sus marcadores favoritos, crear grupos públicos o privados, agregar como amigos a conocidos u otras personas con favoritos interesantes, publicar y recomendar los marcadores en Twitter.

BlinkList, es otro de los sitios más usados que permite utilizar marcadores privados, cuenta con una estética cercana a otros servicios Web 2.0 como Furl y muchas herramientas comunes con Delicious, pero también algunos elementos propios. Está disponible en numerosos idiomas, entre ellos el español, pero las traducciones son ciertamente mejorables y todavía hay muchas lenguas en las que no está disponible.

Uno de los rasgos que definen a Blinklist es su carácter eminentemente social que fomenta la relación entre los usuarios mediante la posibilidad no sólo de definir y almacenar enlaces, sino comentarlos y valorarlos. Este sitio permite mostrar el número de enlaces almacenados, el total de etiquetas, crear crear listas de direcciones que pueden emplearse en la elaboración de una página de inicio rápido, personalizable por el usuario con sus direcciones más habituales y un buscador para acceder al resto de los contenidos almacenados.

Entre las ventajas de Blinklist se pueden destacar las posibilidades para importar los favoritos que el usuario ya tenga almacenados en otros servicios similares, como Delicious o Furl, así como todas las direcciones almacenadas en Internet Explorer, Firefox o cualquier otro navegador que genere listas compatibles. También muestra las nubes de tags como los enlaces dentro de un blog o una página web, mediante un código que el usuario debe copiar. 


\section{El etiquetado social en la blogosfera}

La virtualidad de estos contextos (blogs) ha logrado crear esa conexión que pueda saltar los límites físicos de lo cotidiano y que surjan redes de afinidades donde se estimula la participación entre todos, favoreciendo la comunicación desinhibida y la cooperación. Estas comunidades están basadas en los intereses individuales, las afinidades y los valores de las personas.

Estos sistemas logran visualizar a través de palabras, los rasgos que caracterizan a los usuarios y las comunidades que se generan a partir de determinados intereses comunes. La identidad en la red se construye como una representación de las características reales e imaginarias de los usuarios. Según lo que se construye en estos espacios se está dando a la luz elementos que identifican a los seres humanos en su contexto tradicional. Con el uso de estas plataformas no se delimitan las relaciones entre las personas por el simple hecho de no contar con un contacto físico, al contrario se crean lazos fuertemente sociales como mismo se construyen en el entorno cotidiano.

Las interacciones en los blogs tras el uso de los sistemas de etiquetado social revelan la identidad particular de sus autores, sus intereses y su forma de interpretar la realidad que les rodea. Muchos de los autores de los blogs utilizan términos para definir las etiquetas precisas, puntuales y conocidas por la gran mayoría de los internautas. Estos a su vez brindan a la comunidad una panorámica sobre las principales temáticas que se abordan en los post, pero sin embargo esto no se aplica a toda la comunidad.

Los términos definidos por muchos autores logran acaparar la atención debido a la interpretación del contexto y cómo este es capaz de crear a partir de sus abstracciones etiquetas que son imposibles de comprender por otros. Estas son creadas desde el interés del autor no en función de una comunidad usuaria, las cuales no establecen relación entre los nodos principales de su propia red ni con los generados en la red de otros blogs. Este es un tipo de etiquetado egoísta, donde las etiquetas son definidas sin pensar en la recuperación de sus contenidos. Su interés se enmarca en publicar información que según sus criterios individuales presentan importancia, sin atribuirle valor a las etiquetas como formas de socializar sus publicaciones.

La sociedad ha estado sujeta a diferentes cambios a lo largo de los años, con lo cual, crear un blog que abarque en sus polémicas principales los retos diarios que impone la cotidianidad resulta una tarea interesante y a la vez un gran desafío. En los blogs la mayoría de los autores logran representar su realidad a partir de generalizaciones en sus etiquetas, Política es un ejemplo de esto. La palabra Política es la generalización que abarca los contenidos de los blogs, pero dentro de esta se segmentan otras representaciones tales como: Política Exterior, Política Comercial, etc. que remiten a las principales fuentes de noticias del país del usuario del blog.

Muchos de los términos expuestos por los autores en los blogs, son las particularidades que conforman el diapasón general de su realidad. Las representaciones van encaminadas hacia términos relacionados con: Cultura, Música, Historia, Política, Sexualidad, Género, Discriminación, Personales, Economía, etc., las cuales son capaces de generar otras segmentaciones en cuanto al uso de los términos en la blogosfera, con lo cual se podría afirmar que el comportamiento en cuanto a la definición de los términos y los objetivos perseguidos por el autor para reflejar su realidad en estos medios van encaminados hacia una fragmentación que se extiende de lo general a lo particular.

La definición de etiquetas en estos contextos sociales se produce a partir de estas generalizaciones, las cuales reducen el marco sobre el interés de lo que se desea publicar y recuperar, lo que varía en este caso es precisamente cómo los blogueros interpretan esos contenidos. La interpretación de estos términos generales o particulares estará determinada a raíz de los juicios y criterios sobre esa realidad observable. La conformidad o credibilidad en esta información que se transmite dependerá en gran medida de los portavoces que las emitan y la posición de estos frente a la comunidad bloguera.

La aplicación de un análisis métrico en esta temática de investigación permite identificar una serie de etiquetas que por su comportamiento de uso en la blogosfera son las más representativas. La interpretación de estos resultados cuantitativos posibilita crear los criterios fundamentados que respalden la identificación de los principales escenarios sobre los cuales los autores de los blogs son capaces de construir nuevos conocimientos. Estos contextos están relacionados esencialmente con aquellas temáticas que reportan satisfacciones personales y sociales para la comunidad y determinan en cierta medida la construcción de la cotidianidad. 
Las etiquetas en estos espacios permiten visualizar desde diferentes posiciones la interpretación de una realidad concreta o tergiversada en la cual cada eje será capaz de defender sus propios criterios, y un término que en esencia puede presentar un mismo significado logra avalar otros, debido precisamente a la posición adoptada tras la interpretación de los contenidos publicados y los términos que lo definen. La interpretación de estas etiquetas estará encaminada hacia la posición defendida de cada autor, es por ello que la capacidad de aceptación y entendimiento por parte de las comunidades sobre la representación de la información dependerá en gran medida de los juicios de cada usuario tras lo publicado no basado en la terminología.

A pesar que existan diferencias terminológicas en cuanto a las etiquetas utilizadas en los post, no se puede caracterizar un error en el uso de estas. Cuando la realidad que se describe logra generar diferentes relaciones entre los blogs y presenta seguidores que utilicen los contenidos publicados y estos sean recuperados sustentadas en ellas, estas deben de ser admitidas. La diversidad en los sistemas de etiquetado forma parte de la interpretación de los diferentes contextos, a pesar que a juicios de una gran parte de la sociedad sean considerados sin sentido alguno.

Las etiquetas utilizadas por los autores de los blogs no definen un prototipo específico en su utilización, estas simplemente son definidas tras la interpretación de los contenidos que este realiza, producto del intercambio constante con su medio. El límite de ocurrencias en los blogs esta determinado según el criterio del autor y el interés que este presente de difundir sus contenidos a partir de la recuperación de los términos por otros blogueros.

La gran mayoría de las publicaciones realizadas en los blogs están relacionadas con las principales temáticas del país en cuestión y tienen un alto valor agregado por el autor porque en la gran mayoría de los casos son experiencias vividas. Estas vivencias están enmarcadas en un contexto social (la sociedad) con lo cual el mensaje emitido en sus publicaciones logra ser comprendido por la gran mayoría de la población, a pesar que este sea transmitido de diferentes maneras. La esencia del suceso se logra vislumbrar porque se encuentran relacionados con la cotidianidad, la cual a pesar de estar sujeta a cambios y pueda variar en función de la forma en que esta es observada por el autor, los atributos más generales pueden ser comprendidos, debido que es el mismo contexto con el que se interactúa.

\section{Conclusiones}

Los avances tecnológicos han llevado los procesos de indización a manos de los usuarios en la web, donde la creación de los términos enriquece cada vez más el universo temático, las facilidades de intercambio de información basada en un lenguaje natural, la creación de nuevos conocimientos y productos de valor informativo y social.

El etiquetado social como otro de los servicios de la web 2.0 se ha convertido en esa herramienta práctica, flexible, capaz de representar los contenidos publicados en diferentes plataformas, combinar ideas y puntos de vista que generan intensos debates en torno a disímiles temáticas, crear redes de usuarios, organizar los recursos del blog y localizar de manera más detallada la información

Estos sistemas de etiquetado social permiten la construcción de términos asociados a la realidad, los cuales pueden ser capaces de adoptar varios significados dependiendo de la interpretación, dominio del tema y posición del usuario ante el mismo. Las etiquetas son capaces de definir las características básicas de los objetos que se describen en la web sobre el juicio del autor que los crea, las cuales tras su uso mediante la interacción con los usuarios de la comunidad va tomando un mayor valor agregado entre un universo de categorías.

La asignación de etiquetas en estos contextos presenta una serie de particularidades que la identifican y sientan las bases por su utilidad para desarrollar diversas investigaciones donde no solo se logra vislumbrar los principales contenidos para publicar y sociabilizar en la web a partir de la interacción del autor con la realidad que le circunde, sino también el comportamiento de estos términos en el tiempo, así como el impacto del mismo en un determinado sector de la sociedad. 


\section{BibliصS social tagging: a model for representing information in the blogosphere}

\section{Referencias}

CAÑADA, J. (2006). Tipologías y estilos del Etiquetado Social. Disponible en: http://www. herrero.ugr.es/gbd/docs/tagging.pdf

GOLDER, S. \& HUBERMAN, B. (2006). Usage patterns of collaborative tagging systems. Journal Of Information Science, 32(2).

HERNÁNDEZ, A. (2008). Folksonomías: las más recientes evidencias ecológicas en la industria de la información. Ciencias de la Información, 39(2).

KROSKI, E. (2005). The hive mind: folksonomies and user-based tagging. Diponible en: http://infotangle.blogsome.com/2005/12/07/the-hive-mind-folksonomies-and-user-based-tagging/

MALDONADO, D (2009). Identidad Folksonómica en el hábitat web: interiorización de sus fundamentos con un enfoque transdisciplinar para nuevas practicas y discursos en las Ciencias de la Información. Tesis de Licenciatura no publicada, Facultad de Comunicación. La Habana.

MARLOW, C. (2006). Investment and attention in the weblog community. Disponible en:

http://alumni.media. mit.edu/ cameron/cv/pubs/2006-investment-and-attention-in-the-weblog-community.pdf

NAYA, Z. (2009). ¿Weblogs: revolución digital? La Tecla. Disponible en: http://latecla.cu/bd/digital/weblogs-revolucion-digitalzeus.htm

QUINTARELLI, E. (2005). Folksonomies: power to the people. Disponible en: http://www.iskoi.org/doc/folksonomies.htm

SINHA, R. (2005). A cognitive analysis of tagging. Disponible en: http://www.rashmisinha.com/archives/05 09/taggingcognitive.html 


\section{Datos de los autores}

Elaine Pérez Sanchidrián

Lic. en Bibliotecología y Ciencias de la Información. Especialista en Información Comercial. Ministerio de Comercio Exterior (MINCEX). Centro para la Promoción del Comercio Exterior y la Inversión Extranjera (CEPEC), Cuba. elaine.perez@mincex.cu

\section{Raúl Campos Posada}

Dr. en Ciencias de la Educación y Tecnologías de la Información. Maestro Investigador de la Facultad de Ingeniería Mecánica y Eléctrica. Universidad Autónoma de Coahuila, México.

rcamposposada@hotmail.com

\section{Gloria Elisa Campos Posada.}

Lic. en Sistemas Computacionales y Administrativos. Maestra Investigadora de la Facultad de Ingeniería Mecánica y Eléctrica. Universidad Autónoma de Coahuila, México.

gecamposposada@hotmail.com

Recibido-Received : 2014-07-29

Aceptado - Accepted : 2014-09-30

\section{(cc) $\mathbf{E Y}$}

This work is licensed under a Creative Commons Attribution 4.0

United States License.

\section{ULIS $\underline{D}$ - Sente}

This journal is published by the University Library System of the University of Pittsburgh as part of its D-Scribe Digital Publishing Program and is cosponsored by the University of Pittsburgh Press. 